4. Dunkel IJ, Shi W, Salvaggio K, et al. Risk factors for severe neutropenia following intra-arterial chemotherapy for intra-ocular retinoblastoma. PLoS One. 2014;9(10): e108692.

Disclosures A. Baker: None. S. Ammanuel: None. M. Caton: None. K. Narsinh: None. A. Ashfar: None. A. Banerjee: None. D. Cooke: None. C. Dowd: None. M. Amans: None. V. Halbach: None. R. Higashida: None. S. Hetts: None.

\section{E-072 THE RELATIONSHIP BETWEEN CEREBRAL VASOSPASM AND HERPESVIRUS REACTIVATION AFTER ANEURYSMAL SUBARACHNOID HEMORRHAGE}

${ }^{1} \mathrm{M}$ Walker*, ${ }^{1} \mathrm{~A}$ Mohammed, ${ }^{1} \mathrm{C}$ Kelly, ${ }^{1} \mathrm{~S}$ Levy, ${ }^{2} \mathrm{M}$ Erdoes, ${ }^{3} \mathrm{C}$ Johnston, ${ }^{1} \mathrm{M}$ Levitt. ${ }^{1}$ Neurological Surgery, University of Washington, Seattle, WA; ${ }^{2}$ Convent of the Sacred Heart High School, New York, NY; ${ }^{3}$ Allergy and Infectious Disease, University of Washington, Seattle, WA

\subsection{6/neurintsurg-2021-SNIS.167}

Background Aneurysmal subarachnoid hemorrhage (aSAH) is a devastating disease frequently leading to death or poor functional outcome. A major source of disability from aSAH is the development of cerebral vasospasm, which is defined as narrowing of the large and medium-sized intracranial arteries. Limited information exists regarding underlying anatomic mechanisms of vasospasm after aSAH. Based on the anatomical location of resident herpesvirus and their activation in response to adrenergic stress, we propose that herpesvirus reactivation in response to adrenergic activation of head and neck ganglia during aSAH will be temporally related to cerebral vasospasm.

Methods We developed an IRB-approved protocol for noninvasive bedside testing of viral shedding in tears and saliva in aSAH patients. The protocol was a joint effort with Infectious Disease and our virology laboratory. Viral specimens and catecholamines were obtained at admission and at days 4, 7, 10 and 14 post-aSAH. These values were compared to standardof-care metrics including transcranial doppler, clinical examination and radiological studies. Herpesvirus serology was also obtained.

Results Our protocol successfully yielded samples for analysis in all cases. Initially, serum catecholamines were utilized but collection methodology and requirements resulted in unusable samples. Further, many patients require pressor support using parenteral catecholamines and serum results may not be valid during hospitalization. Instead, salivary alpha-amylase is being tested as a surrogate marker, with collection limited to those patients not requiring catecholamine pressor support within the previous 24 hours. In our preliminary dataset, integrity analyses demonstrated high quality yields of saliva and tears for quantitative PCR analysis. Once collection is complete, we aim to present the collection and storage protocol for dissemination and highlight the training required for specimen processing.

Conclusion We have developed a novel protocol for the analysis of viral shedding and catecholamine measurement in postaSAH patients. The specimens are captured during the patient's hospitalization and allow us to study the relationship between reactivation of chronic herpesvirus infection and cerebral vasospasm after aSAH.

Disclosures M. Walker: 2; C; Medtronic. A. Mohammed: None. C. Kelly: None. S. Levy: None. M. Erdoes: None. C.
Johnston: None. M. Levitt: 1; C; Medtronic, Stryker. 2; C; Medtronic, Metis Innovative. 4; C; eLoupes, Cerebrotech, Synchron.

\section{E-073 CLOT BANK COLLABORATIVE REGISTRY PROTOCOL: NOVEL METHOD FOR EVALUATING ISCHEMIC THROMBUS}

${ }^{1} \mathrm{M}$ Walker*, ${ }^{2} \mathrm{~A}$ Edlund, ${ }^{3} \mathrm{C}$ Dupont, ${ }^{4} \mathrm{G}$ Tan, ${ }^{1} \mathrm{C}$ Kelly, ${ }^{1} \mathrm{~S}$ Levy, ${ }^{1} \mathrm{E}$ Federico, ${ }^{1} \mathrm{M}$ Levitt. ${ }^{1}$ Neurological Surgery, University of Washington, Seattle, WA; ${ }^{2}$ Genomic Medicine Group, J. Craig Venter Institute, La Jolla, CA; ${ }^{3}$ Microbial and Environmental Genomics Group, J. Craig Venter Institute, La Jolla, CA; ${ }^{4}$ Infectious Disease Group, J. Craig Venter Institute, La Jolla, CA

\subsection{6/neurintsurg-2021-SNIS.168}

Background Epidemiological and laboratory studies have brought renewed attention to the myriad ways in which bacteria contribute to risk of stroke and to adverse outcomes after stroke. The emergence of endovascular thrombectomy as a treatment provides a unique opportunity to advance our understanding of infection in stroke patients. Thrombus retrieved from cerebral arteries represents a new source of information providing unprecedented biological insight into the vascular microenvironment. Our aim was to develop a reproducible protocol allowing for the preservation of microbiota and their DNA/RNA during collection and transfer of thrombi and peripheral blood specimens from facilities across remote or distant geographies for future multi-omic analysis.

Methods Utilizing Materials Transfer Agreements with partners in several states, we developed an IRB-approved protocol for collection, archive and study of specimens retrieved during standard-of-care thrombectomy. The protocol was a joint effort among University of Washington stroke physicians, clinical scientists, and multiple laboratories at the J. Craig Venter Institute. Using pre-labeled sterile laboratory supplies in the angiography suite, a step-by-step protocol for specimen retrieval and processing was outlined for interventionalists and their team.

Results Our protocol successfully yielded samples for analysis in all cases. Preliminary metagenomic analysis, including negative controls, demonstrated sufficient DNA quality and quantity for evaluation. We present the tissue preservation protocol for dissemination and highlight the analytical challenges presented by low biomass, high host-contamination specimens. In addition, we discuss the benefits of room (ambient) temperature preservation media compared to others requiring refrigeration or freezing for storage and transport.

Conclusion We have developed a novel tissue banking protocol to preserve microbiota from arterial thrombi retrieved from ischemic stroke patients. While there is no consensus which class of pathogens is implicated in the susceptibility to stroke, multiple studies including our own, have shown bacterial fragments and communities in cerebral thrombi. The specimens captured during standard-of-care thrombectomy allow us to characterize and functionally define the microbiota associated with cerebral thrombi in ischemic stroke patients.

Disclosures M. Walker: 2; C; Medtronic. A. Edlund: None. C. Dupont: None. G. Tan: None. C. Kelly: None. S. Levy: None. E. Federico: None. M. Levitt: 1; C; Medtronic, Stryker. 2; C; Medtronic, Metis Innovative. 4; C; eLoupes, Cerebrotech, Synchron. 